\title{
Handwritten Character Recognition using Neural Network and Tensor Flow
}

\author{
Megha Agarwal, Shalika, Vinam Tomar, Priyanka Gupta
}

\begin{abstract}
The paper will describe the best approach to get more than $90 \%$ accuracy in the field of Handwritten Character Recognition (HCR). There have been plenty of research done in the field of HCR but still it is an open problem as we are still lacking in getting the best accuracy. In this paper, the offline handwritten character recognition will be done using Convolutional neural network and Tensorflow. A method called Soft Max Regression is used for assigning the probabilities to handwritten characters being one of the several characters as it gives the values between 0 and 1 summing up to 1 . The purpose is to develop the software with a very high accuracy rate and with minimal time and space complexity and also optimal.
\end{abstract}

Keywords-Handwritten Character Recognition,

Convolutional Neural Network, Tensor Flow, Soft Max Regression

\section{INTRODUCTION}

Understanding the handwritten characters or typed documents is simple to the human beings as we have the ability to learn. The same ability can be induced to the Machines also by the use of Machine Learning and Artificial Intelligence. The field which deals with this problem is called as the OCR or also known as Optical Character Recognition. It's the area of study among various fields such as recognizing of pattern, also Image vision and also AI. This is the system for changing electronic and image text into the digital character to be read by the machines.

The time used in entering the data and also the storage space required by the documents can be highly reduced by the use of OCR or in other words it can be retrieved fast. By using the OCR in banking field, legal scenarios, etc. many important and sensitive documents can be processed faster without human intervention.

OCR in advance can be inferred in two ways based on type of the text and document acquisition (Figure 1).

Further if we take into consideration the text type, then OCR is further of two types, HCR (Handwritten Character Recognition) which is intelligent recognition of the handwritten text and PCR (Printed Character Recognition). We need the high recognition ability due to the varying handwriting of the humans. Many a times the writing style of same individual is different at times. Further OCR is characterized into two forms as Offline and Online recognition systems based on acquiring of the documents. Offline System deals with recognizing the pre written

Revised Manuscript Received on April 12, 2019.

Megha Agarwal, Computer Science and Engineering, SRM IST Ghaziabad, India. (E-mail: megha.80agr@gmail.com)

Shalika, MCA Department, KIET Group of Institutions Ghaziabd, India. (E-mail: shalika.chhabra20@gmail.com)

Vinam Tomar, Computer Science and Engineering, SRM IST Ghaziabad, India. (E-mail: vinamtomar@gmail.com)

Priyanka Gupta, Computer Science and Engineering, SRM IST Ghaziabad, India. (E-mail: priyanka6gupta@gmail.com) document acquired through various input methods. But in Online recognizing system, the writing is recognized the moment it is written. The device used for the online system is Electric pen where it is used for writing the letters or words on the device called as digitizer and on the basis of the pen movement the input is recorded.

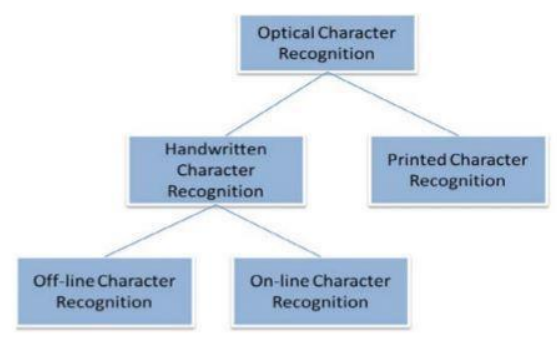

Figure 1. Optical Character Recognition classification

\section{LITERATURE REVIEW}

Research in the region of word recognition, being done from Grimsdale in the year 1959 is soonest endeavor to perceive the handwritten character. This mid-sixty research exhibited the utilization of examination by combination strategy being proposed by the eden in $1968 . \mathrm{He}$ demonstrated that every single handwritten character is limited to number of schematic highlights. This hypothesis was later utilized as a part of almost all strategies for auxiliary methodologies in the region of character recognition.

K. Gaurav and Bhatia P. K [2], proposed different prehandling systems being associated with the recognition of the characters. The procedure took a shot at the various types of pictures from a basic picture-based report to a hued and changed forces including foundation. Different systems of pre-handling and standardization like skew remedy, differentiate evacuation, commotion expulsion and numerous other upgrade procedures were recommended. They reached the decision that a solitary procedure can't be connected for preprocessing the picture. Yet additionally there were a few disparities that utilizing every one of these systems likewise can't give the best exactness comes about.

Salvador España-Boquera [3], The analysts proposed the utilization of hybrid or half plus half concealed markov show (HMM) to perceive the handwritten content in disconnected mode. The optical model's basic part was prepared with markov chain procedure and a multilayer perceptron was likewise used to gauge the probabilities.

Published By:

Blue Eyes Intelligence Engineering \& Sciences Publication 
In [4], to perceive the disconnected handwritten numerals of six prominent Indian language, a changed quadratic classifier is utilized. A similar paper likewise manages perceiving the English letters in order. For both of these, a multilayer perceptron was utilized and Boundary following and Fourier descriptors were utilized for the component extraction. By examining the shape and looking at their highlights, the characters were identified. Also, to decide the quantity of concealed layers, back spread system was utilized. With this very calculation, a recognition rate of 94\% have been accounted for with less preparing time.

R. Bajaj, S. Chaudhari, L. Dey, et al [5], for grouping the Devanagari numerals, distinctive highlights like clear part, thickness and minute highlights were utilized. sAdditionally, to increase the recognition capacity, the paper proposes multi classifier unwavering quality for handwritten Devanagari numerals.

Sandhya Arora in [6], In this paper specifically four highlights like shadow, histogram of chain code crossing point and horizontal line fitting highlights being portrayed. Among these highlights the shadow was registered all around for picture character, the rest three were processed by partitioning the character picture into the distinctive sections. In the one useful execution utilizing the dataset of 4900 examples demonstrated the exactness rate of $90.8 \%$ for Devanagari handwritten characters.

Nafiz Arica at al. [7] This paper gave the technique because of which it was less demanding to maintain a strategic distance from the preprocessing stage along these lines lessening the loss of imperative data. The best one proposed was calculation of capable division. What's more, the different strategies supporting this calculation were utilizing neighborhood maxima and minima, additionally other, for example, stroke tallness which turned out to be ideal and furthermore character limit. What's more, these were altogether connected on a grayscale picture. Utilizing this approach, superfluous division was decreased bit by bit.

Alongside that, the paper additionally proposed another model called shrouded markov demonstrate (HMM) preparing for estimation of worldwide and highlight space parameters alongside estimation of model parameters. Additionally, to rank the individual characters and furthermore to get the shape data, this preparation show was utilized. Additionally, by utilizing the one-dimensional portrayal of a 2-D character picture tremendously builds the energy of HMM for shape perceiving.

In [8], a technique was proposed to perceive the individually Tamil written character by utilizing the grouping in the strokes. Principally a strokes' format or shape-based portrayal is utilized spoken to as a string of shape highlights. Utilizing this strategy, the unrecognized stroke was perceived by contrasting it and a dataset of strokes by the string coordinating method in an adaptable mode. Utilizing this, an individual character was perceived by distinguishing every one of the strokes and its segments.

\section{THE PROPOSED RECOGNITION SYSTEM \& RESULTS}

Normally HCR is categorized into six phases which are acquisition of image, pre-processing of input image, segmentation, feature extraction, classification and post processing. The block diagram of the basic character recognition is shown in figure 2 .

The very first phase, pre-processing deals with the methods necessary to construct the raw input images. The segmentation phase deals with segmenting the raw image into single characters and then arranging it into $m^{*} \mathrm{n}$ pixels to deal with it mathematically. The feature extraction method is perhaps of main significant issue in achieving high recognition percentage for which various techniques like Deformable templates, Gradient feature, Contour profiles, Fourier descriptors, Gabor features, Graph description, Geometric moment invariants, Template matching, Unitary Image transforms, Projection Histograms, Zoning, Zernike Moments and Spline curve approximation are used.

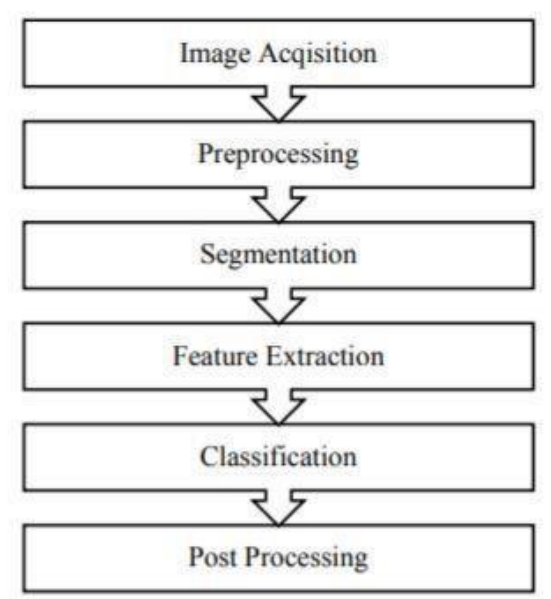

Figure 2. Block diagram of HCR

\section{A. Image Acquisition}

In Image acquisition stage, the input image is provided to the recognition system. The input can be either in an image format such as JPEG, BMT, etc. or scanned image, digital camera or any other suitable digital input device or one can draw on the canvas provided on the user interface.

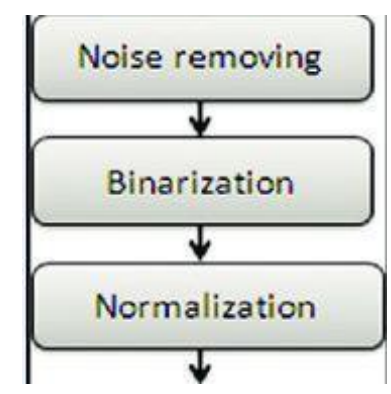

\section{B. Pre-processing}

The second method called as Pre-processing is the entry method for recognition of character and very important in deciding the recognition rate. Preprocessing works to normalize the strokes and also remove variations that can reduce the rate of accuracy. Preprocessing mainly works on the various distortions like the irregular text size, points missed during the pen movement, jitters, left-right bend and uneven spaces. 
1. Noise Removing: To eliminate the unwanted or undesired patterns, a technique called noise removing is used. There's a technique like Uniform and nonuniform filtering that is to be used.

2. Binarization: In this all typed characters are translated into grey-scale picture. Each and every image of character is to be catched vertically after translating the gray scale image into the binary matrix.

3. Normalization: It is the process of translating a picture data into the standard required form. Mainly sizing along with skewing normalizations are performed. Size modifies the picture image in to the predefined fixed size. While skew is used during the time of scanning when the text is deviated from the base line and for this skewing and detections and their backpropagation results are required.

\section{Segmentation}

Segmentation is used to convert input image consisting of many characters into the individual characters. The techniques used are word, line and character segmentation. It is generally performed by dividing single characters from the word picture. Moreover, a content is processed in a way that is tree like. In initial scenario, row histogram is used to segment the lines. Then after, every level, characters are retrieved by technique called histogram and then finally getting it retrieved.

\section{Feature Extraction}

The aim of feature extraction is to allow the extraction of pattern which is most important for the classification. Some of the Feature extraction techniques like Principle Component Analysis (PCA), Scale Invariant Feature Extraction (SIFT), Linear Discriminant Analysis (LDA), Histogram, Chain Code (CC), zoning and Gradient based features can be applied to extract the features of individual characters. All of these features are used to train the given system.

Each of the segmented image is taken of some pixel of dimension $28 * 28$.

This can be performed to be as a big array of numbers.

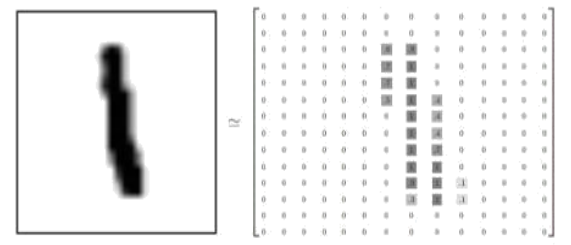

There by flattening the array into a vector of $28 * 28=$ 784 numbers. Thus, the image now converges to a minimal bunch of arrays in a 784-cell dimension of a highly efficient structure. The image now becomes a tensor of ndimensional array.

\section{E. Classification}

The decision making is done in the classification phase. For recognizing the characters, the extracted features are used. Different classifiers like SVM and Neural Networks are used. The classifiers sorts the given input feature with reserved pattern and find the best matching class for input, for which Soft Max Regression is used.
Soft Max regression assigns the probability to each result thus classification becomes easy. It basically first adds up all the evidences it gets by the below formula and then convert that into the possible probabilities

$$
\text { evidence }_{i}=\sum_{j} W_{i, j} x_{j}+b_{i}
$$

Here, weights and bias are denoted by $\mathrm{Wi}$ and bi respectively for the class $i$, and $j$ denoted by summing over the reserved pixels in the input image $x$. We translate the proof of the tallies into the evaluated probabilities ' $y$ ' using the "softmax" which is an activation or link function,

$$
\mathbf{y}=\operatorname{softmax}(\text { evidence) }
$$

Now for converting the probabilities of input being in any of the defined class,

$$
\operatorname{Softmax}(x)=\operatorname{normalize}(\exp (x))
$$

\section{F, Post-Processing}

The final and last phase of the character recognition is the Post-processing. It is the procedure for correcting the misclassified output by using natural language. It processes output by getting it after the shape have been recognized. If the shape is recognized purely then the accuracy can be improved in accordance with the knowledge of language. Shape recognizers behave differently for different handwriting input. For the few, it results in a individual characters of string while also including a few number of alternates in second case, by including the measure of confidence in every alternative

\section{CONCLUSION}

After going through the papers, it was concluded that feature extraction method like diagonal and direction techniques are way better in generating high accuracy results compared to many of the traditional vertical and horizontal methods. Also using a Neural network with best tried layers gives the plus feature of having a higher tolerance to noise thus giving accurate results.

In neural network the model called as feed forward is mainly trained using the back-propagation algorithm so as to classify and recognize the characters as well get trained more and more. Apart from these, use of normalization along with feature extraction yielded the better and higher accuracy results in character recognition. It is also observed that bigger our training data set and better neural network design, the better accurate is the result.

\section{ACKNOWLEDGMENT}

We would like to acknowledge the support of the Department of Computer Science and Engineering, SRM Institute Science and Technology for their guidance and support they have provided especially Ms. Megha Agarwal, Assistant Professor, Computer Science and Engineering. 


\section{REFERENCES}

1. Singh, Sameer, Mark Hewitt,"Cursive Digit And Character Recognition on Cedar Database", Pattern Recognition, 2000.Proceedings. 15th international conference on. Vol. 2. IEEE 2000

2. K. Gaurav and Bhatia P. K., "Analytical Review of Preprocessing Techniques for Offline Handwritten Character Recognition", 2nd International Conference on Emerging Trends in Engineering \& Management, ICETEM, 2013.

3. Salvador España-Boquera, Maria J. C. B., Jorge G. M. and Francisco Z. M., "Improving Offline Handwritten Text Recognition with Hybrid HMM/ANN Models", IEEE Transactions on Pattern Analysis and Machine Intelligence, Vol. 33, No. 4, April 2011.

4. U. Pal, T. Wakabayashi and F. Kimura, "Handwritten numeral recognition of six popular scripts," Ninth International conference on Document Analysis and Recognition ICDAR 07, Vol.2, pp.749-753, 2007.

5. Reena Bajaj, Lipika Dey, and S. Chaudhury, "Devnagari numeral recognition by combining decision of multiple connectionist classifiers", Sadhana, Vol.27, part. 1, pp.-59-72, 2002.

6. Sandhya Arora, "Combining Multiple Feature Extraction Techniques for Handwritten Devnagari Character Recognition", IEEE Region 10 Colloquium and the Third ICIIS, Kharagpur, INDIA, December 2008.

7. Nafiz Arica, and Fatos T. Yarman-Vural, -Optical Character Recognition for Cursive Handwriting, „IEEE Transactions on Pattern Analysis and Machine Intelligence, vol.24, no.6, pp. 801-113, June 2002.

8. K. H. Aparna,, Vidhya Subramanian, M. Kasirajan, G. Vijay Prakash, V. S. Chakravarthy, Sriganesh Madhvanath, "Online Handwriting Recognition for Tamil", IWFHR, 2004, Proceedings. Ninth International Workshop on Frontiers in Handwriting Recognition, Proceedings. Ninth International Workshop on Frontiers in Handwriting Recognition 2004, pp. 438-443, doi:10.1109/IWFHR.2004

9. Surya Nath R S M, Afseena "Handwritten Recognition- A review", International Journal of Scientific and Research Publications, Volume 5, Issue 3, March 20151 ISSN 22503153

10. Anita Pal and Davashankar Singh,"Handwritten English Character Recognition Using Neural Network", International Journal of Computer Science and Communication, pp: 141144, 2011.

11. J.Pradeep1 , E.Srinivasan2 and S.Himavathi3, "DIAGONAL BASED FEATURE EXTRACTION FOR HANDWRITTEN ALPHABETS RECOGNITION SYSTEM USING NEURAL NETWORK" International Journal of Computer Science \& Information Technology (IJCSIT), Vol 3, No 1, Feb 2011

12. Theingi Htike and Yadana Thein "Handwritten Character Recognition Using Competitive Neural Trees " IACSIT International Journal of Engineering and Technology, Vol. 5, No. 3, June 2013

13. Ayush Purohit \#1, Shardul Singh Chauhan\#2 "A Literature Survey on Handwritten Character Recognition" Ayush Purohit et al, / (IJCSIT) International Journal of Computer Science and Information Technologies, Vol. 7 (1) , 2016, 1-5

14. Manoj Sonkusare and Narendra Sahu "A SURVEY ON HANDWRITTEN CHARACTER RECOGNITION (HCR) TECHNIQUES FOR ENGLISH ALPHABETS" Advances in Vision Computing: An International Journal (AVC) Vol.3, No.1, March 2016

15. Hallale, Sumedha B., and Geeta D. Salunke. "Twelve Directional Feature Extraction for Handwritten English Character Recognition." International Journal of Recent Technology and Engineering 2, no. 2 (2013) 